\title{
Preliminary study on the incidence of hot spots in Protected Areas - GEF Terrestre
}

\author{
Israel Benjamim ${ }^{1}$, Marina Amaral ${ }^{2}$, Lawrence Oliveira ${ }^{3}$, Roberto Rivera-Lombardi ${ }^{4}$
}

\author{
${ }^{1}$ University of Brasília (UnB), Brasília, Brazil \\ ${ }^{2}$ Ministry of the Environment (MMA), Brasília, Brazil \\ ${ }^{3}$ Brazilian Institute of Environment and Renewable Natural Resources (Ibama), Brasília, Brazil \\ ${ }^{4}$ Universidad Central de Venezuela (UCV), Caracas, Venezuela \\ *e-mail: israel.benjamim@mma.gov.br; marina.amaral@mma.gov.br; lawrence.oliveira@ibama.gov.br; \\ robertoriveralombardi@gmail.com
}

\begin{abstract}
The GEF-Terrestre is a Federal Government project, aligned with the principles of the Convention on Biological Diversity and the United Nations Framework Convention on Climate Change, which aims to promote the conservation, recovery and management of biodiversity in the Caatinga, Pampa and Pantanal biomes, for through the strengthening of the management of 28 Conservation Units (UCs). One of the lines of action of the GEF-Terrestre is the integrated fire management (MIF) in at least one UC of each biome targeted by the project. In this sense, the objective of this work was to carry out a preliminary study on the incidence of hot spots in the project's UCs in the 2014-2018 period. For this purpose, data from hot spots (reference satellite MODIS / Aqua-Tarde), provided by the National Institute for Space Research (INPE), were used as indicators of burnt areas detected from images obtained by space sensors. Using the QGIS application (3.4.5) the data of hot spots were crossed with the areas covered by the UCs and their surroundings (10 km distance), generating information related to the spatio-temporal distribution of the hot spots. The preliminary results showed that in the UCs there is a higher incidence of hot spots in the surroundings (2.505) than inside them (1.787). Likewise, it should be noted that of the total of the UCs selected by the project (28), eleven (11) UCs did not present any occurrence of hot spots during the study period. Finally, we conclude the need to carry out more in-depth studies using medium spatial resolution satellite images (such as the Landsat and Sentinel 2 satellites) in the units highlighted in this study, in order to assess the dynamics of fire in the studied UCs.
\end{abstract}

Keywords: biodiversity; fire management; burnt areas; monitoring; management 